\title{
MicroRNA-506 participates in pancreatic cancer pathogenesis by targeting PIM3
}

\author{
JUNDONG DU $^{1 *}, \mathrm{XI} \mathrm{ZHENG}^{2 *}$, SHOUWANG CAI ${ }^{3}$, ZIMAN ZHU $^{1}$, \\ JINGWANG TAN ${ }^{1}$, BIN HU $^{1}$, ZHIQIANG HUANG ${ }^{3}$ and HUABO JIAO ${ }^{1}$ \\ Departments of ${ }^{1}$ Hepatobiliary Surgery and ${ }^{2}$ Division Three for Senior Officers, \\ The First Affiliated Hospital to the General Hospital of the PLA; ${ }^{3}$ Department of Hepatobiliary Surgery, \\ The General Hospital of the PLA, Beijing 100853, P.R. China
}

Received July 8, 2014; Accepted March 18, 2015

DOI: $10.3892 / \mathrm{mmr} .2015 .4109$

\begin{abstract}
MicroRNA (miRNA) is a type of short non-coding RNA that suppresses the expression of protein coding genes by partial complementary binding to the 3'-untranslated regions (UTRs) of mRNAs. miRNA expression alterations are involved in the initiation, progression and metastasis of human cancer and it has been suggested that miRNAs function as tumor suppressors as well as oncogenes in cancer development. PIM-3 is a member of the proto-oncogene PIM family, the aberrant expression of which exists in human pancreatic cancer tissues. There are reports indicating that overexpression of PIM3 is associated with the promotion of pancreatic cancer cell proliferation. The aim of the present study was to identify micro (mi)RNAs that regulate the expression of the oncogene PIM3 in PC. It was confirmed that the expression of PIM3 was regulated by miRNAs through an AGO2 knockout experiment. Subsequently, a dual luciferase assay system was constructed and used to screen 13 selected miRNAs that may target the PIM3 3'UTR directly. The results indicated that miR-15a/b, miR-16, miR-33a/b, miR-124, miR-195 and miR-506 repressed the luciferase activity by targeting the PIM3 3'UTR. However, only the expression of miR-506 was negatively correlated with PIM3 expression in PC tissues $(\mathrm{r}=-0.38, \mathrm{P}=0.017)$. Furthermore, a biological functional study indicated that miR-506 functioned as a tumor suppressor by repressing PC cell proliferation, which was partially reversed
\end{abstract}

Correspondence to: Dr Zhiqiang Huang, Department of Hepatobiliary Surgery, The General Hospital of the PLA, 28 Fuxing Road, Beijing 100853, P.R. China

E-mail: zhiqianghuangcn@163.com

Dr Huabo Jiao, Department of Hepatobiliary Surgery, The First Affiliated Hospital to the General Hospital of the PLA, 51 Fucheng Road, Beijing 100853, P.R. China

E-mail: huaboj@163.com

*Contributed equally

Key words: microRNA, PIM3, pancreatic cancer, cell proliferation by PIM3 overexpression. To the best of our knowledge, the present study was the first to reveal the tumor suppressor function of miR-506 in PC, which has the potential to be employed in the diagnosis and treatment of PC.

\section{Introduction}

Pancreatic cancer (PC) is an aggressive malignancy with one of the highest mortality rates amongst cancers worldwide. It is the sixth leading cause of mortality from malignant disease in China and the fourth leading cause of cancer-associated mortality in the USA (1-3). Rapid tumor progression, late diagnosis, early and aggressive metastasis and high resistance to conventional chemotherapy lead to exceptionally poor prognosis with an overall five-year survival rate of $<5 \%$ (4). Therefore, novel markers for early diagnosis and novel therapeutic targets for PC require to be identified. Although the etiology of PC is also attributed to numerous environmental factors, the accumulation of genetic and epigenetic changes remains the fundamental mechanism of tumorigenesis (5-7).

MicroRNA (miRNA/miR) is a type of short non-coding RNA that suppresses the expression of protein-coding genes by partial complementary binding, particularly to the 3'-untranslated regions (3'UTRs) of mRNAs. miRNA expression alterations are involved in the initiation, progression, and metastasis of human cancer and it is believed that miRNAs function as tumor suppressors and oncogenes in cancer development $(8,9)$. Accumulating studies have shown that disturbed expression of miRNAs is involved in the process of pathogenesis and drug resistance $(10,11)$.

PIM3 was initially identified as a novel gene that is induced by membrane depolarization or forskolin in the rat pheochromocytoma cell line PC12 and was designated as a kinase induced by depolarization (KID)-1 (12). However, KID-1 was renamed PIM3 because it showed high sequence similarity with the proto-oncogenic provirus integrating site Moloney murine leukemia virus (PIM) family of proteins (13). Recently, PIM3 was found to be aberrantly expressed in pancreatic ductal adenocarcinoma (PDAC) cells and to phosphorylate the pro-apoptotic protein B-cell lymphoma 2-associated death promoter (14). In addition, PIM3 was shown to be regulated by transcription factors such as ETS-1 and serve as a positive 
regulator of signal transducer and activator of transcription 3 signaling in PC cells $(15,16)$.

The present study aimed to investigate the post-transcriptional regulation of the expression of PIM3 in PC, focusing on miRNAs directly targeting PIM3.

\section{Materials and methods}

Cell culture. PANC-1, MIAPaca-2 and HEK293T cells (China Infrastructure of Cell Line Resources, Beijing, China) were cultured in Dulbecco's modified Eagle's medium (DMEM) containing $10 \%$ fetal bovine serum (Hyclone, Logan, UT, USA), $100 \mathrm{IU} / \mathrm{ml}$ penicillin and $10 \mathrm{mg} / \mathrm{ml}$ streptomycin (Hyclone). All cells were maintained at $37^{\circ} \mathrm{C}$ under an atmosphere of $5 \% \mathrm{CO}_{2}$.

Tissue samples. PC and matched adjacent normal tissues from 38 patients were obtained post-operatively from March to September in 2012 from the Department of Heptapobiliary Surgery, The First Affiliated Hospital to the General Hospital of the PLA (Beijing, China). The patients provided signed, informed consent for their tissues to be used for scientific research. Ethical approval for the study was obtained from the First Affiliated Hospital to the General Hospital of the PLA. Diagnoses were based on pathological and/or cytological evidence. Histological features of the specimens were evaluated by two senior pathologists according to classification criteria from the World Health Organization (17). Tissues were obtained from patients prior to chemotherapy or radiation therapy. Specimens were immediately frozen and stored at $-80^{\circ} \mathrm{C}$ prior to western blot and reverse transcription quantitative polymerase chain reaction (RT-qPCR) analyses.

Western blot analysis. Protein extracts were boiled in SDS/ $\beta$-mercaptoethanol sample buffer (Sigma-Aldrich, St. Louis, MO, USA), and $30 \mu \mathrm{g}$ of each sample was loaded onto a lane of a $12 \%$ polyacrylamide gel (Sigma-Aldrich). The proteins were separated by electrophoresis, and the proteins in the gels were blotted onto polyvinylidene difluoride membranes (GE Healthcare, Little Chalfont, UK) by electrophoretic transfer. The membrane was incubated with rabbit anti-PIM3 monoclonal antibody (1:1,000; ab75776; Abcam, Cambridge, MA, USA), mouse anti- $\beta$-actin monoclonal antibody $(1: 1,000$; sc-58673; Santa Cruz Biotechnology, Inc., Dallas, TX, USA) or rabbit anti-AGO2 monoclonal antiobdy (1:1,000; ab186733; Abcam) for $1 \mathrm{~h}$ at $37^{\circ} \mathrm{C}$. The specific protein-antibody complex was detected by using horseradish peroxidase-conjugated goat anti-rabbit (sc-2004) or rabbit anti-mouse (sc-358920) immunoglobulin G (1:5,000; Santa Cruz Biotechnology, Inc.). Detection by enhanced chemiluminescence (ECL) reaction was carried using an ECL kit (Pierce Biotechnology, Rockford, IL, USA) and X-ray films (Carestream Health, Inc., Xiamen, China). The $\beta$-actin signal was used as a loading control. The band intensity was analyzed by using Quantity One software, version 4.6.2 (Bio-Rad Laboratories, Inc., Hercules, CA, USA).

RT-qPCR analysis. RT-qPCR analysis was used to determine the relative expression levels of 13 selected miRNAs. Total RNA was extracted from tissues, using TRIzol (Invitrogen Life Technologies, Carlsbad, CA, USA) according to the
A
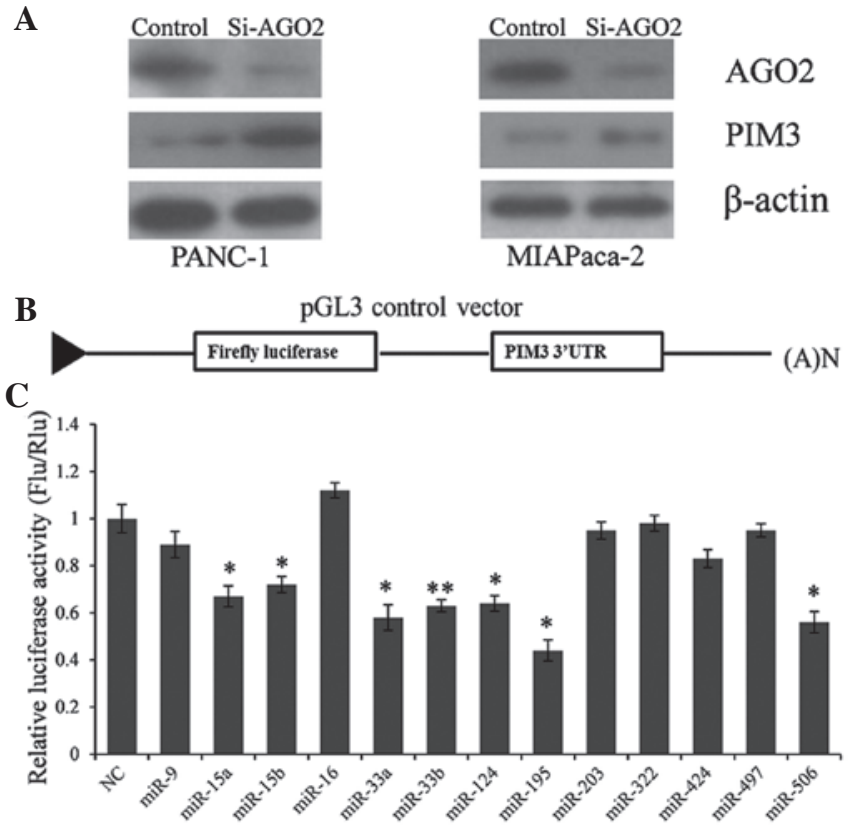

Figure 1. Expression of PIM3 is repressed by miRNAs in pancreatic cancer cells. (A) The pancreatic cancer cell lines PANC-1 and MIAPaca-2 were transfected with AGO2 siRNA and corresponding control. $48 \mathrm{~h}$ after transfection, cells were lysed and the expression of AGO2 and PIM3 was detected by western blot analysis with $\beta$-actin as the loading control. (B) Schematic diagram for inserting the full-length PIM3 3'UTR into the pGL3 control vector. (C) Dual luciferase assay to screen miRNAs that target the PIM3 3'UTR directly. The results were analyzed using Student's t-test and the results are expressed as the mean \pm standard deviation; ${ }^{*} \mathrm{P}<0.05,{ }^{* *} \mathrm{P}<0.01$. si, small interfering; UTR, untranslated region; AGO2, argonaute RNA-induced silencing complex catalytic component 2 ; miR, microRNA; NC, negative control; Flu/Rlu, firefly luciferase light unit/Renilla luciferase light unit.

manufacturer's instructions. The expression levels of candidate miRNAs were detected by TaqMan miRNA RT-Real Time PCR (Applied Biosystems Life Technologies, Foster City, CA, USA). Single-stranded cDNA was synthesized by using the TaqMan MicroRNA Reverse Transcription kit (Applied Biosystems Life Technologies, Waltham, MA, USA) and then amplified by using TaqMan Universal PCR Master Mix (Applied Biosystems Life Technologies) together with miRNA-specific TaqMan Minor Groove Binder probes (Applied Biosystems Life Technologies). U6 small nuclear RNA was used for normalization. The experiments were processed using an ABI 7300 PCR Thermal Cycler (Applied Biosystems Life Technologies). The protocol for qPCR was a classic two step PCR: $95^{\circ} \mathrm{C}$ for $10 \mathrm{~min} ; 95^{\circ} \mathrm{C}$ for $15 \mathrm{sec}$ followed by $60^{\circ} \mathrm{C}$ for $1 \mathrm{~min}$ for 35 cycles. Each sample in each group was measured in triplicate and the experiment was repeated at least three times. The relative expression was calculated using the $\Delta \Delta \mathrm{Ct}$ method. The products were separated by $2 \%$ agarose (Sigma-Aldrich) to confirm the specificity of the PCR reaction.

3'UTR luciferase reporter assays. To generate the 3'UTR luciferase reporter, the full-length 3'UTR from PIM3 was cloned into the downstream region of the firefly luciferase gene in the pGL3-control vector (Promega, Madison, WI, USA). The primer sequences for PIM 3'UTR cloning were as follows: PIM3, forward CTCGAGGGAGCTGCACCTG ACTGGGA and reverse TCTAGATATGTACAAAAACA 

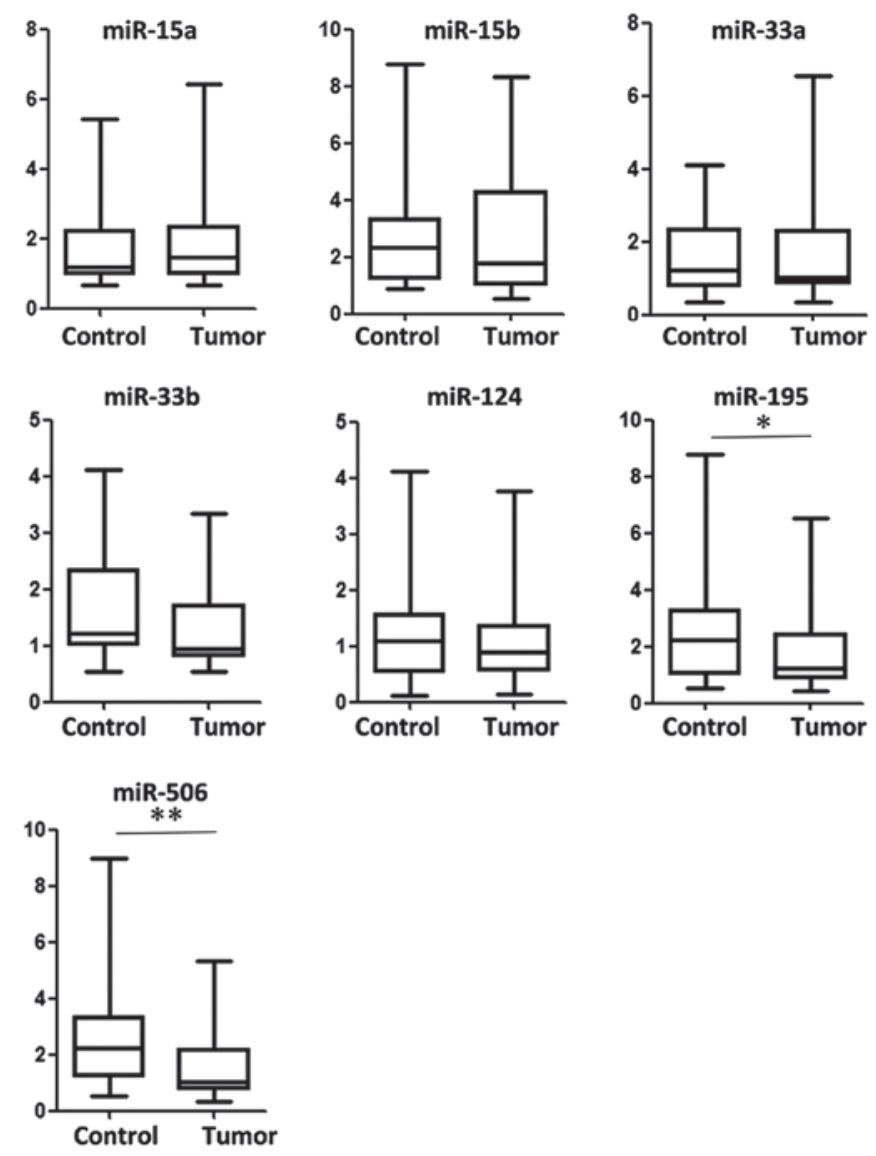

Figure 2. Detection of the expression of selected miRNAs in pancreatic cancer tissues. Total RNA was extracted using TRIzol reagent and the expression of seven selected miRNAs was detected by reverse transcription polymerase chain reaction analysis. U6 small nuclear RNA was used for normalization. Each sample in each group was measured in triplicate and the experiment was repeated at least three times. Results were expressed with box-and-whisker plots where the boxes represent $95 \%$ confidence interval and the bars represents the range of the data. ${ }^{*} \mathrm{P}<0.05,{ }^{* *} \mathrm{P}<0.01$. miR, microRNA.

TTTTAATTGAAATACC. The primers were synthesized by BGI-GBI Biotech Co., Ltd. (Beijing, China). miRNA mimics and inhibitor were synthesized by GenePharma Co., Ltd (Shanghai, China). The sequence for the double strand miR-506 mimic was 5'-UAAGGCACCCUUCUGAGU AGA-3', for the single strand miR-506 inhibitor was 5'-TCT ACTCAGAAGGGTGCCTTA-3', for the miR-506 control was 5'-UUCUCCGAACGUGUCACGUTT-3' and for the miR-506 inhibitor control was 5'-CAGUACUUUUGUGUA GUACAA-3'. The pRL-TK vector (Promega) containing Renilla luciferase was co-transfected for data normalization. For luciferase reporter assays, HEK293T cells were seeded in 48-well plates. Luciferase reporter vectors were co-transfected with one of the miRNA mimics by using Lipofectamine 2000 (Invitrogen Life Technologies). Two days post-tranfection, cells were harvested and assayed using the Dual-Luciferase Assay system (Promega). Each treatment was performed in triplicate in three independent experiments. The results were expressed as relative luciferase activity (firefly luciferase light units/Renilla luciferase light units). To identify the binding site of miR-506, a plasmid with three nucleotide mutations in the predicted miR-506 binding site was used.

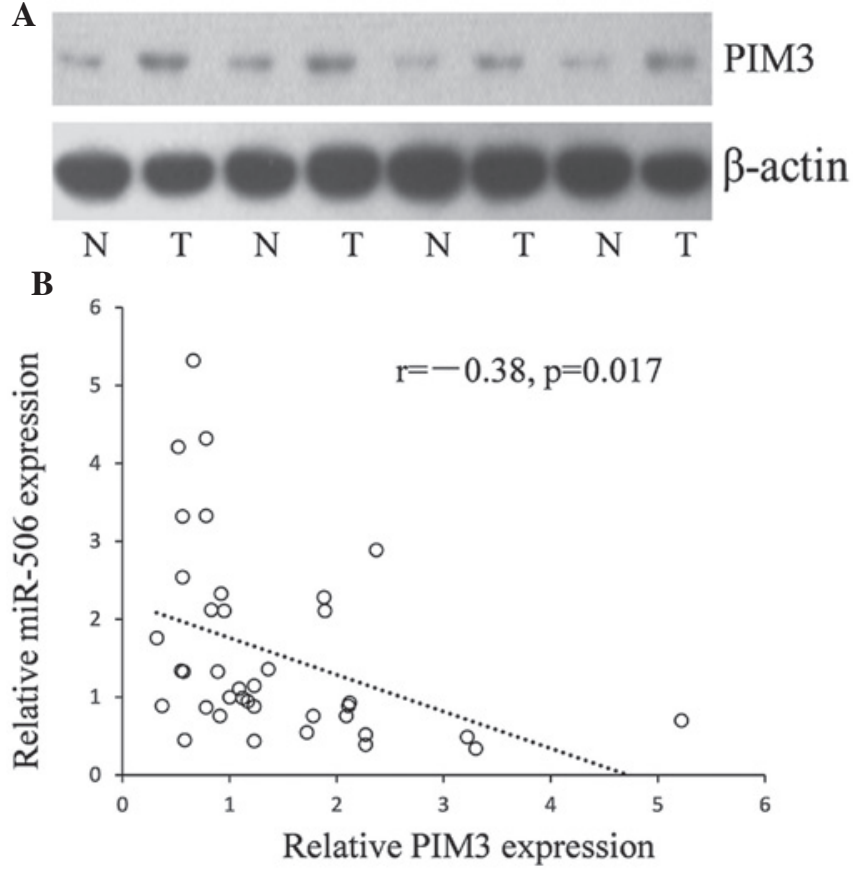

Figure 3. Negative correlation between the expression of miR-506 and PIM3. (A) Representative western blots showing PIM3 expression in pancreatic cancer tissues and adjacent normal tissues. To evaluate PIM3 expression, the relative band intensity was determined by using Quantity One software with the expression of $\beta$-actin for normalization. (B) Correlation between miR-506 and PIM3 in 38 clinical samples of pancreatic cancer indicated an inverse correlation (Pearson correlation, $\mathrm{r}=-0.38 ; \mathrm{P}=0.017$ ). $\mathrm{N}$, normal tissue; $\mathrm{T}$, tumor tissue; miR, microRNA.

AGO2 knockdown. Pancreatic cancer cell lines PANC-1 and MIAPaca-2 were transfected with AGO2 siRNA and the corresponding control using Lipofectamine 2000 (Invitrogen Life Technologies). A total of $48 \mathrm{~h}$ subsequent to transfection, the cells were lysed by RIPA Lysis and Extraction Buffer (Pierce Biotechnology) and the expression of AGO2 and IPM3 was detected by western blot analysis. $\beta$-actin was used as loading control.

Cell proliferation assay. PANC-1 and MIAPaca-2 cells were seeded in 96-well plates at a low density $\left(5 \times 10^{3}\right)$ in DMEM culture and allowed to attach overnight. The cells were then transfected with miR-506 mimic or inhibitor, with a scrambled-sequence single strand or double strand short hairpin RNA as the control. MTT ( $20 \mu \mathrm{l} ; 5 \mathrm{mg} / \mathrm{ml}$; Sigma-Aldrich) was added into each well $48 \mathrm{~h}$ after transfection, and the cells were incubated for a further $4 \mathrm{~h}$. Following addition of dimethyl sulfoxide (Sigma-Aldrich), the absorbance was measured at $570 \mathrm{~nm}$ using a 96-well plate reader.

Statistical analysis. Data were analyzed by using SPSS Statistical Package version 17 (SPSS, Inc., Chicago, IL, USA). Results were analyzed using Student's t-test. $\mathrm{P}<0.05$ was considered to indicate a statistically significant difference between values. The results of the luciferase and MTT assays were displayed as the mean \pm standard deviation. The results of miRNA expression in the clinical samples were exhibited using box-and-whisker plots, where the whiskers represented the range of the data. 

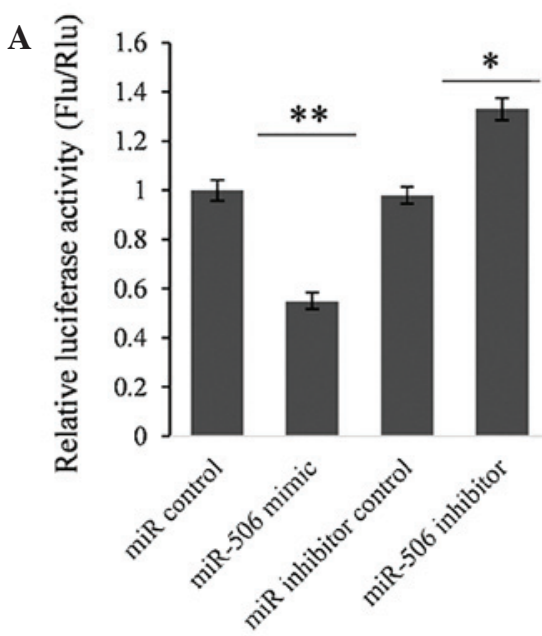

C

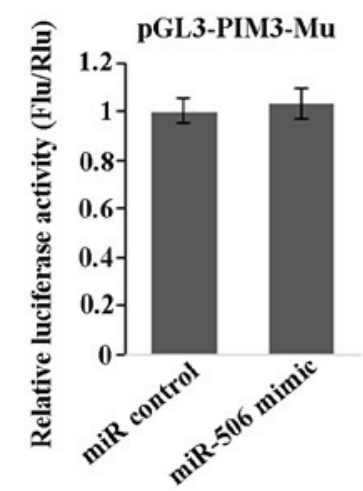

B

Position 141-147 of wild type PIM3 3'UTR 5' ...UGACCUUCGCUUUGAGUGCCUUU... Hsa-miR-506 3, AGAUGAGUCUUCCCACGGAU Position 141-147 of mutant PIM3 3'UTR 5 ' ...UgaccuUcgcuUUgagugccuUU... Hsa-miR-506 3, AgAugagucuUCCGACAAAAU

D
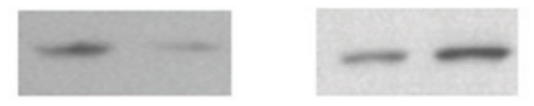

PIM3

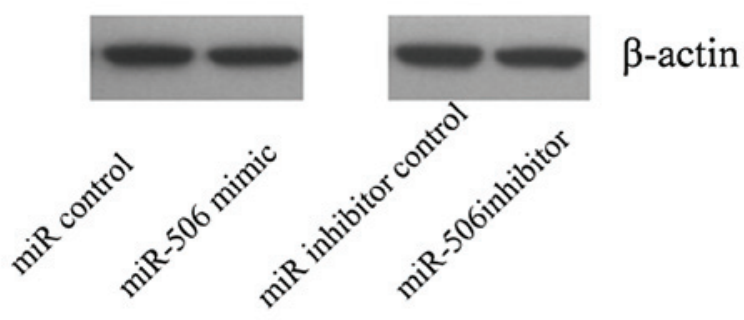

Figure 4. PIM3 is a target gene of miR-506. (A) HEK293T cells were co-transfected with miRNA control, miR-506 mimic, anti-miR control or miR-506 inhibitor in a dual-luciferase assay. (B) Schematic diagram for the predicted interaction between miR-506 and PIM3 mRNA. Red characters represent the mutant nucleotides. (C) A dual luciferase assay was employed to confirm the binding site of miR-506. (D) PIM3 protein levels of PANC-1 cells transfected with miR-506 mimic or miR-506 antagonist were detected by western blot analysis. The results were analyzed using Student's t-test and the results are expressed as the mean \pm standard deviation; ${ }^{*} \mathrm{P}<0.05,{ }^{* *} \mathrm{P}<0.01$. miR, microRNA; Wt, wild-type; Mu, mutant; UTR, untranslated region; Flu/Rlu, firefly luciferase light unit/Renilla luciferase light unit.

\section{Results}

PIM3 expression in PC cells is regulated by miRNAs. To explore whether the expression of PIM3 is regulated by miRNAs, argonaute RNA-induced silencing complex (RISC) catalytic component 2 (AGO2), the key component of the RISC complex was knocked down in PANC-1 and MIAPaca-2 cells. It was observed that the inactivation of the RISC complex caused a marked upregulation of PIM3 expression (Fig. 1A). The result indicated that miRNAs participate in the inverse control system of PIM3 expression.

Five out of thirteen selected miRNAs target PIM3 directly. To identify which miRNAs repress PIM3 expression directly, a reporter vector containing the full-length PIM3 3'UTR downstream of the firefly luciferase coding region was constructed (Fig. 1B). miRNAs that may target the PIM3 3'UTR were predicted using the online bioinformatics tool TargetScan (http://www.targetscan.org), and thirteen miRNAs were selcted: miR-15a, miR-15b, miR-16, miR-33a, miR-33b, miR-124, miR-195, miR-203, miR-322, miR-424, miR-497 and miR-506. After screening by using the dual luciferase system, we seven miRNAs (miR-15a, miR-15b, miR-33a, miR-33b, miR-124, miR-195 and miR-506) were identified to repress luciferase activity by significantly targeting the PIM3 3'UTR (Fig. 1C).

Association between miRNAs and PIM3 expression. To explore the association between PIM3 and miRNAs in PC samples, PIM3 expression was detected by western blot analysis and the expression of seven selected miRNAs was detected by RT-qPCR (Fig. 2). miR-195 and miR-506 were significantly downregulated in tumor tissue samples compared with normal adjacent tissues $(\mathrm{P}<0.05$ and $\mathrm{P}<0.01$, respectively). An example of PIM3 expression in PC and paired normal control tissues is shown in Fig. 3A. The results indicated that $74 \%$ (29/38) of PC tissues displayed upregulated PIM3 expression and $71 \%$ (27/38) or 63\% (23/38) had downregulated miR-506 or miR-195 expression, respectively (Fig. 3B).

To reveal the correlation between downregulated miRNAs (miR-195 and miR-506) and the PIM3 in PC tissues, PIM3 expression was assessed using western blot analysis. An inverse correlation was identified between the expression levels of miR-506 and PIM3 in 38 clinical samples of PC. Low levels of miR-506 were associated with high PIM3 expression (Pearson correlation, $r=-0.38$; $\mathrm{P}=0.017$; Fig. 3B). 
A

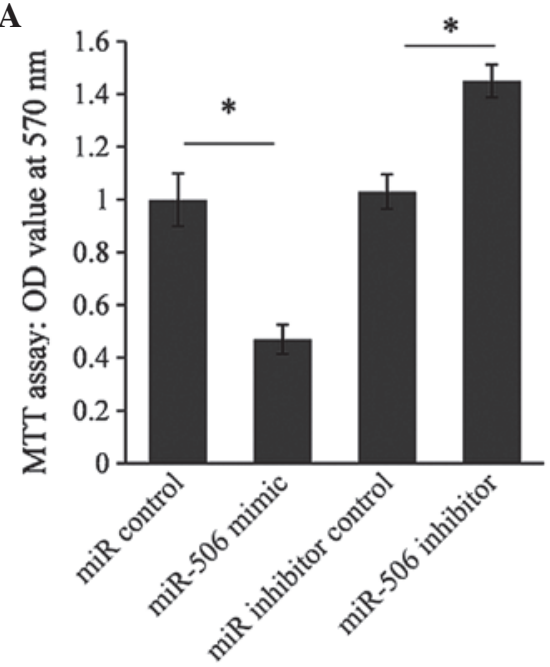

B

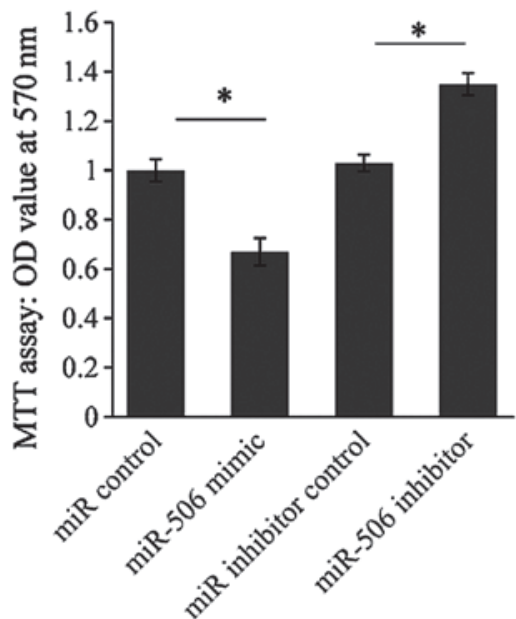

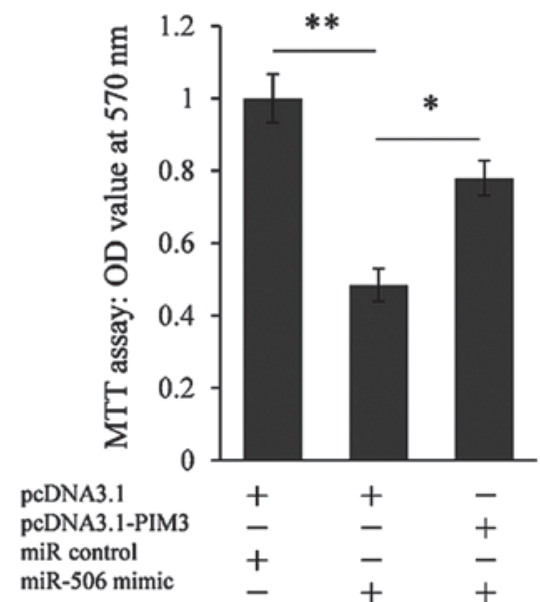

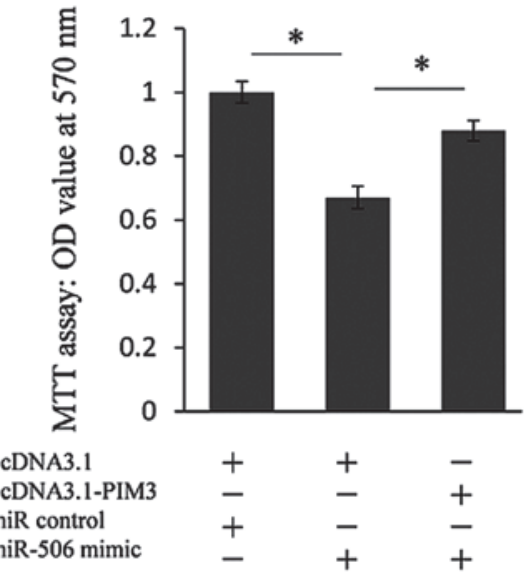

Figure 5. miR-506 suppresses pancreatic cancer cell proliferation by targeting PIM3. (A) PANC-1 or (B) MIAPaca-2 cells were seeded in 96-well plates at low density $\left(5 \times 10^{3}\right)$ and allowed to attach overnight. The cells were then transfected with miR-506 mimics or inhibitor, with a scrambled-sequence short hairpin RNA as the control. An MTT assay was performed $48 \mathrm{~h}$ post transfection. The PIM3 expression vector was co-transfected with miR-506 to identify whether miR-506 disturbs pancreatic cancer cell proliferation partially through PIM3. The results were analyzed using Student's t-test and the results are expressed as the mean \pm standard deviation; ${ }^{*} \mathrm{P}<0.05,{ }^{* *} \mathrm{P}<0.01$. miR, microRNA; OD, optical density.

miR-506 represses PIM3 expression by directly targeting its $3^{\prime} U T R$. To further confirm whether PIM3 is the target gene of miR-506, the dual luciferase assay system was utilized again. HEK293T cells were co-transfected with pGL3-PIM3 and miR-506 mimic or inhibitor. As shown in Fig. 4A, compared with the miRNA control, the miR-506 mimic significantly suppressed the luciferase activity by $45.2 \%(\mathrm{P}<0.01)$. Furthermore, the luciferase activity was significantly upregulated (by $35.7 \%$ ) by the miR-506 inhibitor compared with that in the miR-inhibitor control $(\mathrm{P}<0.05)$. These changes of firefly luciferase translation indicated that miR-506 targets the 3'UTR of PIM3.

A seed sequence mutation clone was also used to further confirm the binding site for miR-506 (Fig. 4B). A vector containing a putative miR-506 binding region in the $3^{\prime} \mathrm{UTR}$ of PIM3 with three mutant nucleotides (designated as pGL3-PIM3-Mu) was constructed. The bar graph in Fig. 4C shows that the enzyme activity was not significantly reduced in cells transfected with miR-control compared with that in cells transfected with miR-506 mimic $(\mathrm{P}>0.05)$. This result indicated that miR-506 may suppress PIM3 expression through binding to the seed sequence at the 3'UTR of PIM3.
To further examine whether endogenous PIM3 expression is suppressed by miR-506, PANC-1 cells were transfected with miR-506 mimic or inhibitor. PIM3 protein levels were detected by western blot analysis $48 \mathrm{~h}$ post-transfection. Compared with the corresponding control, the levels of PIM3 protein were significantly suppressed by miR-506 mimic and upregulated by miR-506 inhibitor in PANC-1 cells (Fig. 4D). These results indicated that miR-506 repressed endogenous PIM3 expression in PC cells by directly targeting the PIM3 3'UTR, and PIM3 is a target gene of miR-506.

miR-506 suppresses PC cell proliferation. To further test whether miR-506 may execute tumor-suppressive functions by targeting PIM3, the effect of miR-506-mediated cell proliferation was assessed using an MTT assay on PANC-1 (Fig. 5A) and MIAPaca-2 cells (Fig. 5B). The cell proliferation ability was significantly reduced by the miR-506 mimic (by 52.8\%) in PANC-1 cells and by $33.1 \%$ in MIAPaca- 2 cells. Furthermore, cell proliferation was significantly upregulated by the miR-506 inhibitor (by $43.2 \%$ ) in PANC-1 cells and by $32.3 \%$ in MIAPaca- 2 cells. To further confirm whether miR-506 
represses cell proliferation by targeting PIM3, a PIM3 expression vector was co-transfected with miR-506 mimic into PANC-1 and MIAPaca-2 cells. As shown in Fig. 5A and B (right), the PIM3 expression vector partially reversed the repressed cell proliferation caused by $\mathrm{miR}-506$ overexpression, indicating that miR-506 suppresses PC cell proliferation partially through targeting PIM3.

\section{Discussion}

PIM3 is a member of the proto-oncogenic PIM family that encodes serine/threonine kinases, and is aberrantly expressed in human PC. Studies have indicated that overexpression of PIM3 is associated with enhanced PC cell proliferation (18). In the present study, it was confirmed that the expression of PIM3 is regulated by miRNAs through an AGO2 knockout experiment. Subsequently, a dual luciferase assay system was constructed and used for screening 13 selected miRNAs that may target the PIM3 3'UTR directly according to a TargetScan analysis. The results indicated that $\mathrm{miR}-15 \mathrm{a} / \mathrm{b}$, miR-16, miR-33a/b, miR-124, miR-195 and miR-506 repressed luciferase activity by targeting the PIM3 3'UTR. However, only the expression of miR-506 was negatively correlated with PIM3 expression in the PC tissues $(r=-0.38, \mathrm{P}=0.017)$. Furthermore, a mechanistic study indicated that miR-506 acted as a tumor suppressor by repressing PC cell proliferation and its anti-proliferative function can be partially reversed by PIM3 overexpression.

Biological functions of miR-506, particularly in cancer, have been studied; however, the roles of miR-506 in carcinogenesis are yet to be elucidated $(19,20)$. In breast, cervical and ovarian cancer, miR-506 is confirmed to be a tumor suppressor through targeting Ki-67, Gli3, CDK4 and CDK6 (20-22). Furthermore, downregulation of miR-506 in cervical cancer was identified to be associated with the cancer pathogenesis (20). However, miR-506 overexpression was also reported in lung cancer and melanoma; however, they appear to have opposite functions. Yin et al (23) reported that miRNA-506 was upregulated in lung cancer patients and its overexpression selectively killed lung cancer cells through inhibiting nuclear factor- $\kappa \mathrm{B}$ p 65 to evoke the generation of reactive oxygen species and p53 activation. By contrast, Streicher et al (24) reported that the miRNA-506-514 cluster was overexpressed in almost all melanoma samples that were assessed and had a positive role in initiating melanocyte transformation and promoting melanoma growth. The present study was the first, to the best of our knowledge, to report that miR-506 was downregulated in PC tissues, which may be applicable for clinical diagnosis. However, since one miRNA may have tens or hundreds of target genes and its function may be tissue-specific, further studies are required to fully unveil the biological functions of miR-506.

\section{References}

1. Li D, Xie K, Wolff R and Abbruzzese JL: Pancreatic cancer. Lancet 363: 1049-1057, 2004.
2. Guo $X$ and Cui Z: Current diagnosis and treatment of pancreatic cancer in China. Pancreas 31: 13-22, 2005.

3. Siegel R, Naishadham D and Jemal A: Cancer statistics, 2012. CA Cancer J Clin 62: 10-29, 2012.

4. Jemal A, Siegel R, Xu J and Ward E: Cancer statistics, 2010. CA Cancer J Clin 60: 277-300, 2010.

5. Tang S, Bonaroti J, Unlu S, et al: Sweating the small stuff: MicroRNAs and genetic changes define pancreatic cancer. Pancreas 42: 740-759, 2013.

6. van Kampen JG, Marijnissen-van Zanten MA, Simmer F, van der Graaf WT, Ligtenberg MJ and Nagtegaal ID: Epigenetic targeting in pancreatic cancer. Cancer Treat Rev 40: 656-664, 2014.

7. Wood LD and Hruban RH: Genomic landscapes of pancreatic neoplasia. J Pathol Transl Med 49: 13-22, 2015.

8. Wu WK, Lee CW, Cho CH, et al: MicroRNA dysregulation in gastric cancer: a new player enters the game. Oncogene 29: 5761-5771, 2010.

9. Nicoloso MS, Spizzo R, Shimizu M, Rossi S and Calin GA: MicroRNAs-the micro steering wheel of tumour metastases. Nat Rev Cancer 9: 293-302, 2009.

10. Ikenaga N, Ohuchida K, Mizumoto K, et al: MicroRNA-203 expression as a new prognostic marker of pancreatic adenocarcinoma. Ann Surg Oncol 17: 3120-3128, 2010.

11. Izumchenko E, Chang X, Michailidi C, et al: The TGF $\beta$-miR200-MIG6 pathway orchestrates the EMT-associated kinase switch that induces resistance to EGFR inhibitors. Cancer Res 74: 3995-4005, 2014.

12. Feldman JD, Vician L, Crispino M, et al: KID-1, a protein kinase induced by depolarization in brain. J Biol Chem 273: 16535-16543, 1998.

13. Konietzko U, Kauselmann G, Scafidi J, et al: Pim kinase expression is induced by LTP stimulation and required for the consolidation of enduring LTP. EMBO J 18: 3359-3369, 1999.

14. Li YY, Popivanova BK, Nagai Y, Ishikura H, Fujii C and Mukaida N: Pim-3, a proto-oncogene with serine/threonine kinase activity, is aberrantly expressed in human pancreatic cancer and phosphorylates bad to block bad-mediated apoptosis in human pancreatic cancer cell lines. Cancer Res 66: 6741-6747, 2006.

15. Li YY, Wu Y, Tsuneyama K, Baba T and Mukaida N: Essential contribution of Ets-1 to constitutive Pim-3 expression in human pancreatic cancer cells. Cancer Sci 100: 396-404, 2009.

16. Chang M, Kanwar N, Feng E, et al: PIM kinase inhibitors downregulate STAT3 (Tyr705) phosphorylation. Mol Cancer Ther 9: 2478-2487, 2010.

17. Jass JR, Sobin LH and Watanabe H: The World Health Organization's histologic classification of gastrointestinal tumors. A commentary on the second edition. Cancer 66: 2162-2167, 1990.

18. Liu B, Wang Z, Li HY, Zhang B, Ping B and Li YY: Pim-3 promotes human pancreatic cancer growth by regulating tumor vasculogenesis. Oncol Rep 31: 2625-2634, 2014.

19. Yang FQ, Zhang HM, Chen SJ, Yan Y and Zheng JH: MiR-506 is down-regulated in clear cell renal cell carcinoma and inhibits cell growth and metastasis via targeting FLOT1. PloS One 10: e0120258, 2015.

20. Wen SY, Lin Y, Yu YQ, et al: miR-506 acts as a tumor suppressor by directly targeting the hedgehog pathway transcription factor Gli3 in human cervical cancer. Oncogene 34: 717-725, 2015.

21. Liu G, Sun Y, Ji P, et al: MiR-506 suppresses proliferation and induces senescence by directly targeting the CDK4/6-FOXM1 axis in ovarian cancer. J Pathol 233: 308-318, 2014.

22. Arora H, Qureshi R and Park WY: miR-506 regulates epithelial mesenchymal transition in breast cancer cell lines. PLoS One 8: e64273, 2013.

23. Yin M, Ren X, Zhang X, et al: Selective killing of lung cancer cells by miRNA-506 molecule through inhibiting NF- $\kappa$ B p65 to evoke reactive oxygen species generation and p53 activation. Oncogene 34: 691-703, 2015.

24. Streicher KL, Zhu W, Lehmann KP, et al: A novel oncogenic role for the miRNA-506-514 cluster in initiating melanocyte transformation and promoting melanoma growth. Oncogene 31: 1558-1570, 2012. 